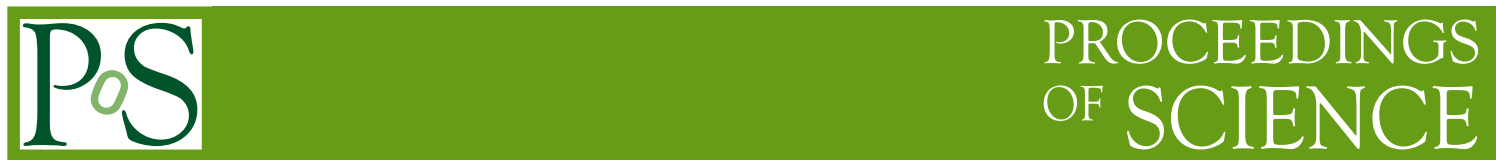

\title{
Higgs searches at ATLAS
}

\author{
Simone PAGAN GRISO* \\ On behalf of the ATLAS Collaboration \\ Lawrence Berkeley National Laboratory, USA \\ E-mail: spagangriso@lbl.gov
}

On July 2012, the ATLAS and CMS collaboration announced together the observation of a new boson in the Higgs searches, with a mass around $126 \mathrm{GeV}$. Since then, the Large Hadron Collider continued to run steadily, providing proton-proton collisions at $\sqrt{s}=8 \mathrm{TeV}$; both experiments have now more than doubled the amount of data available. I will review the latest results from the ATLAS collaboration on Higgs searches, both in the Satandard-Model framework and beyond. I will focus on the updated results with respect to summer 2012, with particular attention to the changes in analysis and calibration techniques; the first results on 2012 data of Higgs searches in the $b$-quarks and tau leptons final states will be presented as well.

LHC on the March

20-22 November 2012

Institute for High Energy Physics, Protvino, Moscow region, Russia

\footnotetext{
*Speaker.
} 


\section{Introduction: the observation of a new boson}

On July 2012 the ATLAS and CMS collaborations jointly announced the discovery of a new particle as a result of the searches for the Standard Model (SM) Higgs boson [1], 2]. The published results used around $11 \mathrm{fb}^{-1}$ of proton-proton collisions data collected at $\sqrt{s}=7 \mathrm{TeV}$ and $8 \mathrm{TeV}$; the combination of the results from the $H \rightarrow Z Z^{(\star)}, H \rightarrow \gamma \gamma$ and $H \rightarrow W^{(\star)} W^{(\star)}$ searches showed an excess of observed events over the expected background. The excess is compatible with the SM Higgs boson expectation, with a p-value exceeding five standard deviations.

Since then the effort of the ATLAS Collaboration has been focused on:

1. strengthen the evidence of the observed signal and measure the production cross section of the new boson;

2. include results of the searches in other decay channels in order to constraint its couplings;

3. measurement of its mass and other properties from angular analysis of its decay products;

4. model-independent searches for beyond SM Higgs boson(s); whenever the search sensitivity could benefit from specific signatures, model-dependent searches were also performed.

For a review of the present status of the mass and properties measurements of the new boson, I invite the reader to refer to Ref [3]. The progress achieved on the other points will be discussed in this contribution, in Section 2, 3 and 4, after a brief overview of the theoretical context and experimental setup. This contribution will focus on the main changes and updates since the summer 2012 results were presented. I refer the reader to the conference notes of each of the presented analyses for a more detailed description on each of them [诃.

\subsection{Theoretical framework}

The expected production cross section and decays branching ratios for the Higgs boson in the SM framework are determined once the Higgs boson mass is set. For a Higgs mass of $\sim 125 \mathrm{GeV}$, the Higgs boson is expected to be produced primarily via the gluon fusion process at the LHC at $\sqrt{s}=7$ or $8 \mathrm{TeV}$; vector-boson fusion production is suppressed by roughly one order of magnitude and the production rate for $W H$ or $Z H$ is even smaller, although the leptonic decays of the $W / Z$ can help to suppress the background. The main decay channels are to a pair of $b$-quarks, and to a pair of $W$ or $Z$ boson; the decays to a pair of photons or $\tau$ leptons are suppressed but offer a particularly favorable signal over background ratio.

Significant theoretical work over the last decade has improved the accuracy of the production cross section predictions, reaching nex-to-next leading logarithm (NNLL) accuracy in QCD and next-to-leading order (NLO) accuracy in electro-weak corrections; this level of accuracy allows systematic uncertainty from the inclusive cross section predictions not to be a dominant source of systematic uncertainty in establishing the consistency of the observed production cross section with the expected one. More recent work is focused on the effect of such higher order corrections to the kinematic of the produced boson, in order to estimate the uncertainty in the modeling of key quantities which are used in the experimental search (e.g. the Higgs transverse momentum 
spectrum, the invariant mass of jets produced in association to it, and so on). I refer the interested reader to Ref [ [, , 5] for further details on these topics.

In addition, I would like to mention that the extended usage of full NLO event generators has also been a key factor to reduce theoretical uncertainties in the modeling of signal and background processes.

\subsection{Experimental setup}

The ATLAS detector [\$] is a multi-purpose particle physics detector with approximately forwardbackward symmetric cylindrical geometry. The inner tracking detector (ID) has a pseudo-rapidity coverage of $|\eta|<2.5$ and consists of a silicon pixel detector, a silicon micro-strip detector, and a transition radiation tracker. The ID is surrounded by a thin superconducting solenoid that provides a $2 \mathrm{~T}$ axial magnetic field. A high-granularity lead/liquid-argon (LAr) sampling calorimeter measures the energy and the position of electromagnetic showers within $|\eta|<3$.2. LAr sampling calorimeters are also used to measure hadronic showers in the end-cap $(1.5|\eta|<3.2)$ and forward $(3.1<|\eta|<4.9)$ regions, while an iron/scintillator tile calorimeter measures hadronic showers in the central region $(|\eta|<1.7)$. The muon spectrometer (MS), which surrounds the calorimeters, consists of three large superconducting air-core toroid magnets (each with eight coils), a system of precision tracking chambers $(|\eta|<2.7)$, and fast tracking chambers for triggering. A three-level trigger system selects events to be recorded for offline analysis.

During the data-taking in 2012, all the components of the ATLAS detector were working very efficiently. All major sub-detectors were working properly for $\sim 94 \%$ of the recorded data. The total integrated luminosity delivered by the LHC to ATLAS at $\sqrt{s}=8 \mathrm{TeV}$ had exceeded $18 \mathrm{fb}^{-1}$ when this talk was presented, in addition to the already collected $\sim 5 \mathrm{fb}^{-1}$ of $\sqrt{s}=7 \mathrm{TeV}$ data in 2011. The increase of integrated luminosity delivered during 2012 has come with the cost of a higher number of interactions in the same bunch crossing $(\mu)$, which is also referred to as pile-up. The values of $\mu$ in 2012 have quite a broad distribution ranging from 5 to 40 , with an average value of $\mu=20$. These harsh conditions, often exceeding the design specifications of the experiment $(\mu \simeq 25)$, triggered a lot of developments in refined calibration and analysis techniques to avoid loss of performance in basic object reconstruction and, ultimately, loss of sensitivity for the physics data analyses. Some of the improvements implemented more recently to cope with these conditions will be mentioned when relevant in the following sections.

\section{Strengthen the evidence}

The most sensitive Higgs searches that led to the discovery of the new boson [1] focus on the expected decays of the Higgs boson to a pair of $Z$ bosons, $W$ bosons and photons.

The most recent update of the search for $H \rightarrow W^{\star} W^{\star}$ [ [8] looks for the subsequent decays of the $\mathrm{W}$ bosons into electrons and muons using $13 \mathrm{fb}^{-1}$ of data collected in 2012. Due to severe background from Drell-Yan production, only the $e^{ \pm} \mu^{\mp}$ final state is considered. This update search focuses on the expected signature from an Higgs produced via gluon fusion with a mass in the rage between $90 \mathrm{GeV}$ and $200 \mathrm{GeV}$. Significant missing transverse energy is required to suppress residual Drell-Yan and multi-jet backgrounds. Events are then categorized depending on the number of reconstructed anti- $k_{T}$ jets with with $p_{\mathrm{T}}>25(30) \mathrm{GeV}$ for $|\eta|<2.5(2.5<|\eta|<4.5)$; 

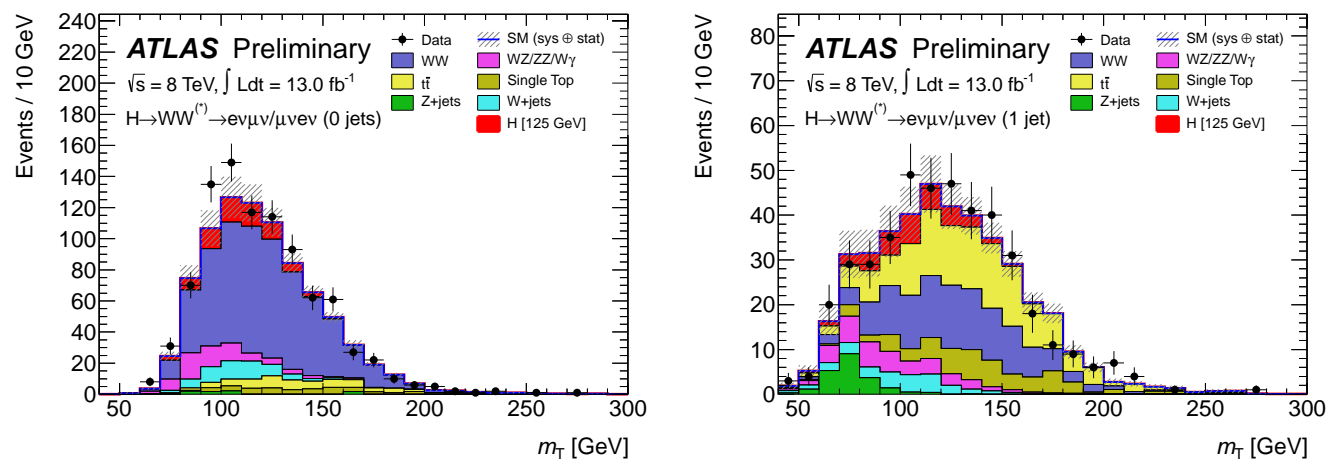

Figure 1: Observed and expected $m_{T}$ distribution for the analyzed $13 \mathrm{fb}^{-1}$ data in the $H \rightarrow W W$ search. The left (right) plot shows selected events with zero (one) jet in the final state. See text for details. Ref [8].

events with no such jets are required to have a transverse momentum of the lepton pair greater than $30 \mathrm{GeV}$ and azimuthal angle difference between the lepton pair system and the missing transverse energy greater than $\pi / 2$. Events with exactly one jet are required not to have any jet tagged as originating from a $b$-quark in order to reduce contamination from $t \bar{t}$ and single top production. To reduce the background contribution from $Z \rightarrow \tau \tau$ with both $\tau$ decaying leptonically, events are vetoed if the reconstructed mass from the di-lepton system and the missing transverse energy, in the hypothesis of collinear $\tau$ decays, is near the $Z$ mass. Events with two or more jets are analyzed separately, being particularly sensitive to vector-boson fusion production, and no update has been released on this analysis at this time. All the selected events are then required to have invariant mass of the lepton pair below $50 \mathrm{GeV}$ and their azimuthal angle difference $\Delta \phi(l l)<1.8$, in order to separate non-resonant $W^{ \pm} W^{ \pm}$production from the Higgs production. These requirements are derived assuming the Higgs boson is a scalar particle, which leads to a correlation in the direction of the leptons in the decay products. The residual background from jets mis-identified as leptons and from non-prompt leptons is estimated with a data-driven technique. The leading background contribution comes from non-resonant $W W$ production and is estimated from the observed yield in a dedicated control region; the extrapolation to the signal region is performed using simulation. A similar technique is also employed to normalize $Z \rightarrow \tau \tau$, and top quark induced background. The normalization obtained with this procedure is always within $13 \%$ of the estimate obtained solely using simulation. The final discriminant used to search for a Higgs boson signal is the transverse mass of leptons and missing energy, defined as: $m_{T}=\sqrt{\left(E_{T}^{\ell \ell}+E_{T}^{\text {miss }}\right)^{2}+\mid \mathbf{p}_{T}^{\ell \ell}+\left(\mathbf{E}_{T}^{\text {miss }}\right)^{2}}$. The observed distribution of $m_{T}$ for the selected events is shown in Figure 1, together with the expected signal and background contributions. A binned likelihood fit on this distribution is used to search for the production of the Higgs boson in the selected events. The observed distribution shows an excess of events over the background-only hypothesis. The probability that a background fluctuation is more signal-like than the observed data $\left(p_{0}\right)$ is found to be $0.3 \%$ for a $125 \mathrm{GeV}$ Higgs boson, corresponding to 2.8 standard deviations of a normal distribution; the production cross section, normalized to the expected Higgs production cross section, is found to be $\mu=\sigma / \sigma_{S M}=1.5 \pm 0.6$, in agreement with the expectation from the SM.

The search for the Higgs boson decaying to a pair of $Z$ bosons and the subsequent decays to 

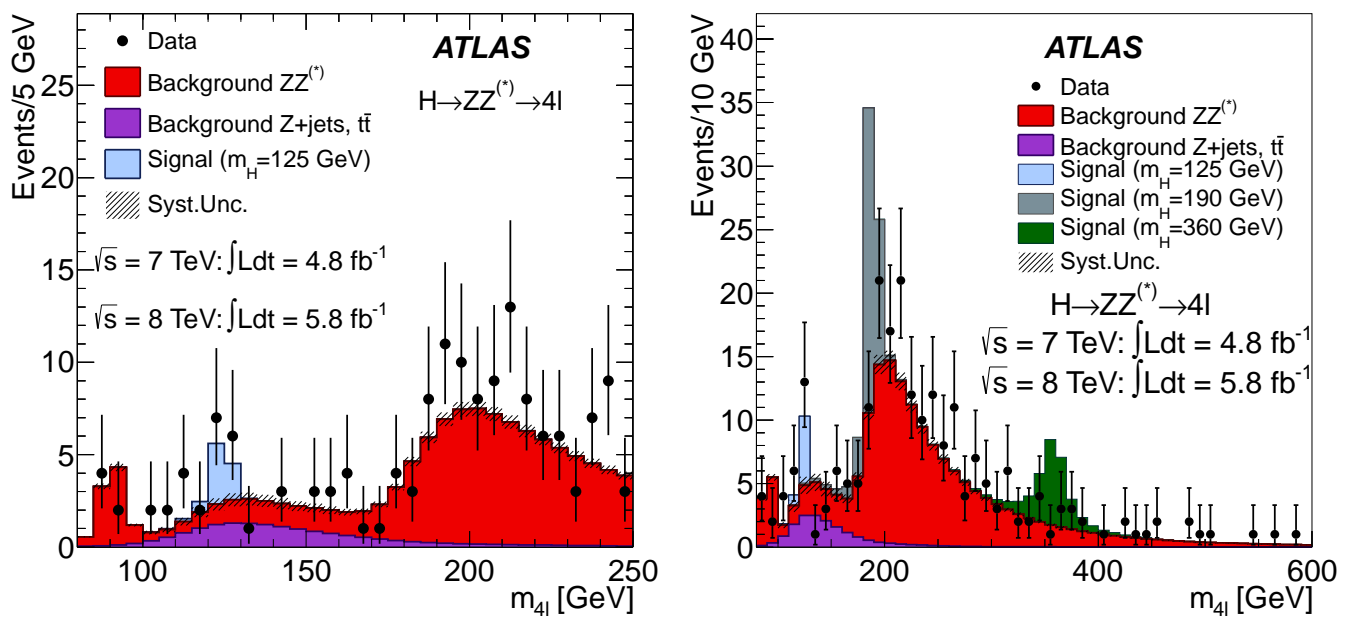

Figure 2: Observed and expected four leptons invariant mass $(m(4 l))$ distribution in the $H \rightarrow Z Z$ search. The left plot shows events with $80<m(4 l)<250 \mathrm{GeV}$, while the right plot extends the $m(4 l)$ range up to $600 \mathrm{GeV}$. The signal expectation for several Higgs mass $\left(m_{H}\right)$ hypotheses is also shown. Ref [1]].

four charged leptons (electrons or muons) has not been updated since summer 2012.The analysis [1] selects events with four charged leptons in the final state, either electrons or muons, with transverse momentum as low as 7(6) GeV for the lower $p_{\mathrm{T}}$ electron (muon). Events are required to have two pairs of oppositely charged and same-flavor leptons with the invariant mass of one pair consistent with an on-shell $\mathrm{Z}$ boson and the other pair consistent with a possible off-shell $Z$ boson. The main background for this analysis comes from non-resonant $Z Z$ production; Top production and $Z+b \bar{b}$ production also contribute significantly. The four leptons invariant mass is used to search for a narrow resonance compatible with the experimental resolution (within 2.1 and $2.8 \mathrm{GeV}$, depending on the decay channel). The observed distribution for the $\sim 11 \mathrm{fb}^{-1}$ of 2011 and 2012 data analyzed so far is shown in Figure 2. An excess of events over the expected background is observed around $m_{H} \sim 125 \mathrm{GeV}$, with a $p_{0}$ equivalent to 3.6 standard deviations and a SM normalized cross section of $\mu=1.2 \pm 0.6$.

The search for the $H \rightarrow \gamma \gamma$ decay channel has not been updated since summer 2012 as well. The analysis [1] selects events with two high- $p_{\mathrm{T}}$ photons of 40 and $30 \mathrm{GeV}$ respectively, with $|\eta|<2.37$. Events are triggered by a di-photon trigger and are categorized depending on the number of jets in the final state, the $\eta$ and $p_{\mathrm{T}}$ of the candidate photon and di-photon system and the presence of detected photon conversions. The main background for this search comes from nonresonant $\gamma \gamma$ production, while $\gamma+$ jets and di-jet production are expected to contribute less than $25-30 \%$ of the total number of selected events. One of the key components of this analysis resides in the photon identification itself, which needs to achieve the best resolution possible to increase separation of signal over the expected non-resonant background, while being stable against the high pile-up environment. The expected resolution for the invariant mass of the di-photon system $(m(\gamma \gamma))$ is expected to range between 3.2 and $6.1 \mathrm{GeV}$ (being on average $3.9 \mathrm{GeV}$ ) and is dominated by the energy resolution of the photons. Background contribution is estimated with a fit of the $m(\gamma \gamma)$ sidebands using an analytic model which has been carefully checked using simulation. The 

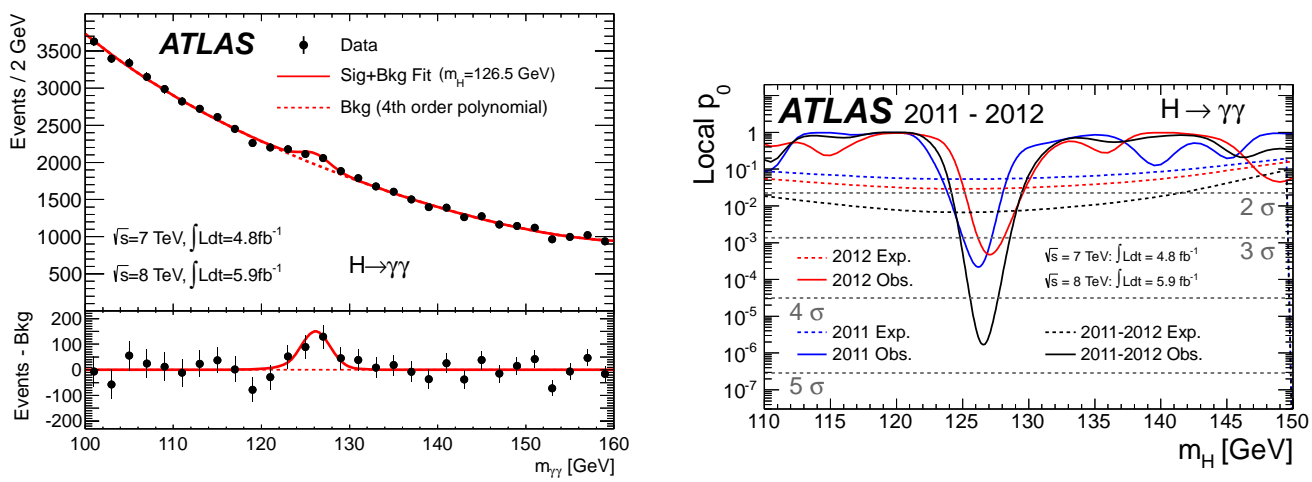

Figure 3: (Left) Observed distribution of $m(\gamma \gamma)$ in the search for $H \rightarrow \gamma \gamma$ decay. The result of the fit in the signal plus background hypothesis is also shown. (Right) $p_{0}$ value as function of the Higgs mass hypothesis, see text for more details. Ref [1].

same fit is used to search for a narrow resonance compatible with the expected signature from a SM Higgs boson that decays to two photons. Overall $11 \mathrm{fb}^{-1}$ of data have been analyzed to search for a narrow resonance on top of the expected non-resonant background. An excess of events over the expected background is found for $m(\gamma \gamma)=126.5 \mathrm{GeV}$ with a $p_{0}$ equivalent to 4.5 standard deviations and a normalized cross section of $\mu=1.8 \pm 0.5$. The observed $m(\gamma \gamma)$ distribution and the $p_{0}$ values as function of the Higgs mass hypothesis are shown in Figure 3 .

\section{Probing new decay channels}

The observation of the newly discovered boson in additional decay channels is a strong test of the expected couplings of the SM Higgs boson. Recently the searches in the associated Higgs production with $W / Z$ boson and subsequent $H \rightarrow b \bar{b}$ decay, and in the inclusive $H \rightarrow \tau \tau$ searches have been updated with the 2012 data sample, and have significantly improved the search sensitivity.

The final state involving a pair of $\tau$ can test the coupling of the new boson to leptons. New results from the $H \rightarrow \tau \tau$ search have been released recently [9], with a significantly improved analysis of the data collected during 2011 and a first analysis of the data collected in 2012 so far, corresponding to a total luminosity of $17.6 \mathrm{fb}^{-1}$. This analysis selects events based on the decay products of the $\tau$ lepton being probed. If the $\tau$ lepton decays to an electron or muon and neutrinos, it will be denoted as $\tau_{\ell}$; if the the $\tau$ lepton decays to one or more charged hadrons it will denoted as $\tau_{h}$. Three main analyses can be then distinguished based on the final state: $\tau_{\ell} \tau_{\ell}$, $\tau_{\ell} \tau_{h}$ and $\tau_{h} \tau_{h}$. After a specific preselection based on the expected final state, events are separated into categories with different signal over background expectation. A first category, targeting vector boson fusion production, is obtained by selecting events with at least two jets in the final state with tight $|\Delta \eta(j j)|$ and $m(j j)$ requirements, in order to enhance contribution from this production mechanism. A so-called boosted category requires large Higgs momentum, reconstructed using visible decay products and missing transverse energy information, in order to target gluon fusion Higgs production; the requirement on the reconstructed Higgs candidate $p_{\mathrm{T}}$ allows to substantially reduce the expected background contribution. In addition few more categories with lower expected 


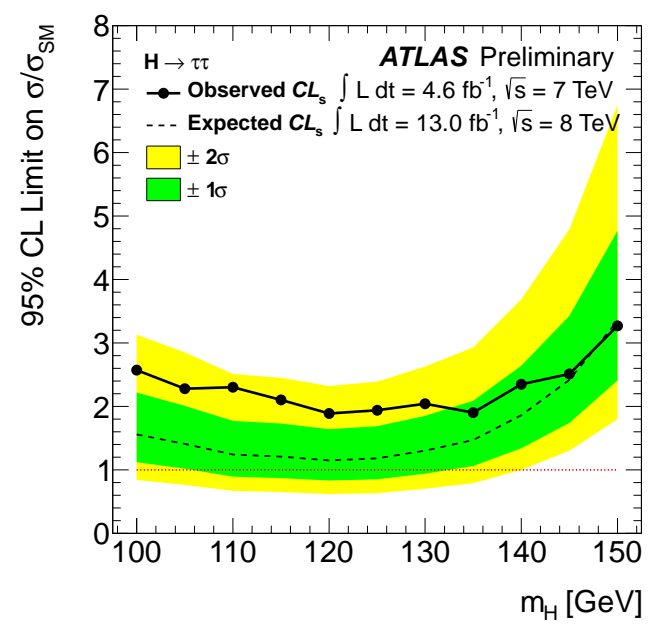

Figure 4: Observed (solid) and expected (dashed) 95\% confidence level upper limits on the Higgs boson cross-section times branching ratio (for the $H \rightarrow \tau \tau$ search), normalized to the SM expectation, as a function of the Higgs boson mass. Expected limits are given for the scenario with no signal. The bands around the

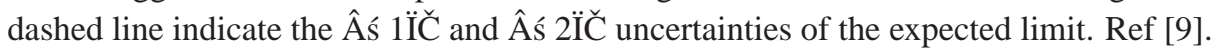

sensitivity are defined both as control regions for the analysis as well to maximize the acceptance of the expected Higgs signal.

The main backgrounds for this analysis come from Drell-Yan production with decay of the vector boson to a pair of $\tau$ leptons, from jets faking $\tau$ and from top quark production. The reconstruction of the Higgs mass is performed by exploiting the expected kinematic correlations between the visible decay products of the $\tau$ leptons and the missing transverse energy observed in the event, achieving between $13 \%$ to $20 \%$ relative mass resolution for a $125 \mathrm{GeV}$ Higgs boson signal.

One of the main improvements with respect to the previous result was a re-newed hadronic $\tau$ identification. A boosted decision tree combining calorimeter and tracking observables have been trained to retain high efficiency and stable performance with the increasing pile-up. The probability for a jet to fake an hadronic $\tau$ has been measured in data from $W$ and $Z$ decays. In addition the energy calibration has been refined in order to reduce the systematic uncertainties on its determination to the level of 3 to $5 \%$, which is a key uncertainty that limits the sensitivity of this search.

A binned likelihood fit on the reconstructed Higgs mass of the candidate events is used to search for the SM Higgs production with several Higgs mass $\left(m_{H}\right)$ hypotheses. No excess of events over the expected background has been observed and 95\% confidence level (C.L.) limits on the production cross section are set; the expected limit for $m_{H}=125 \mathrm{GeV}$ is 1.2 times the expected SM cross section $\left(\sigma_{S M}^{H}\right)$, while the observed limit is $1.9 \sigma_{S M}^{H}$, for the combination of all the $\tau \tau$ search channels. The expected and observed limits for various Higgs mass hypotheses are shown in Figure 4 .

Another powerful search to probe the expected Higgs couplings to fermions has been updated recently. This analysis looks for an Higgs boson that is produced in association with a $W$ or $Z$ boson and the Higgs boson decays to a pair of $b$-quarks [10]. Events are analyzed separately depending on the number of final state leptons (electrons or muons) in the event: zero, one or 

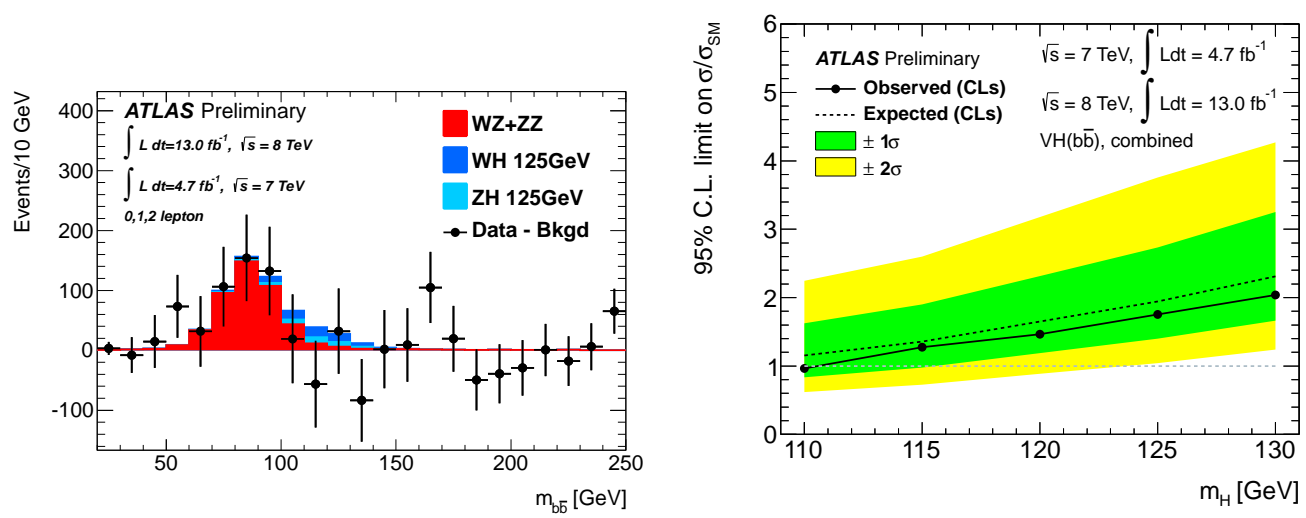

Figure 5: (Left)The $m(b b)$ distribution in data after subtraction of all backgrounds except diboson processes including SM Higgs boson from WH and ZH associated production. The MC backgrounds are normalized according to the results of the global fit. Only statistical uncertainties are shown. (Right)Expected (dashed) and observed (solid) CLs limit on the normalized signal strength as a function of $m_{H}$ for all channels for the combination of the $7 \mathrm{TeV}$ dataset and the $8 \mathrm{TeV}$ dataset. Ref [10].

two. In addition events are categorized in bins of the reconstructed $p_{\mathrm{T}}$ of the associated vector boson, given that high values of such transverse momentum offer a more favorable signal over background ratio. A missing transverse energy trigger is used for events with no leptons in the final state, while for the other two channels single and di-lepton lepton triggers are employed. Exactly two jets reconstructed and $b$-tagged are required, with transverse momentum greater than 45 and $20 \mathrm{GeV}$ respectively. The $b$-tagging of jets is performed using multivariate techniques by exploiting the presence of secondary vertices reconstructed inside the jets, and its performance has been measured in data using $t \bar{t}$ events, leading to a reduction in the uncertainty with respect to what was previously available.

The main backgrounds for this analysis come from $W / Z+j e t s$ and top production. Dedicated control regions are used to normalize such backgrounds, while the extrapolation to the signal region is performed using simulation. The flavor composition of the $W / Z+$ jets background is also constrained in data from a simultaneous fit in all the three channels.

The final search for the Higgs boson production is performed using a binned likelihood fit on the invariant mass of the two $b$-tagged jets: $m(b b)$. In order to test the whole analysis the measurement of the di-boson processes $W Z / Z Z \rightarrow b \bar{b}+X$ has been performed as well. The observed number of events after background has been subtracted is shown in Figure 5 (left). The measured cross section for $W Z / Z Z$ production in such final state is compatible with the expectation from the SM: $\mu_{\text {Diboson }}=\sigma / \sigma_{S M}=1.09 \pm 0.30$ and the observed signal has a significance of 4.0 standard deviations. This measurement is particularly important since the observed SM process has a cross section only five times higher than the one expected from the Higgs production in such decay channels. The search for the Higgs production in the same $m(b b)$ spectrum yields no excess of data over the expected background; the expected 95\% C.L. limit on the Higgs production is 1.9 times the SM cross section $\left(\sigma_{S M}^{H}\right)$ and the observed limit is set to 1.8 times $\sigma_{S M}^{H}$, for a mass of $m_{H}=125 \mathrm{GeV}$, as shown in Figure 5 (right).

In addition a dedicated search for the Higgs produced in association to $t \bar{t}$ and decaying to 
a pair of $b$-quarks has been performed using the $7 \mathrm{TeV}$ dataset [11], corresponding to $4.7 \mathrm{fb}^{-1}$. This search used events with high missing transverse energy and 4 to 6 jets in the final state, out of which up to $4 b$-tagged. A kinematic fit is used to assign jets to the top and Higgs decays, whenever possible. The invariant mass of the two jets associated with the Higgs boson and the sum of the high- $p_{\mathrm{T}}$ objects in the event $\left(H_{\mathrm{T}}\right)$ are used to search for signal contribution. This analysis is not yet sensitive to the expected SM cross section and observed no excess on top of the expected background. The observed 95\% C.L. limits range between 8.5 and 30 times the expected SM cross section, for and Higgs mass hypothesis ranging between 110 and $140 \mathrm{GeV}$.

\section{Look for other signals of Higgs-like particles}

In addition to the presented analyses targeting a SM-like Higgs boson, several other searches have been performed for signatures beyond the ones predicted by the SM. Additional Higgs-like signatures are searched for in the same decay channels presented before up to $m_{H}$ of $600 \mathrm{GeV}$. In addition other final state add significant sensitivity in this mass hypothesis range, including $H \rightarrow Z Z \rightarrow \ell \ell v v, H \rightarrow \ell \ell j j$ and $H \rightarrow \ell v j j$. In all these searches the observed events are compatible with the expected background and limits on the production cross section of such an Higgs boson are set as function of its mass hypothesis [12, 13, 14]. The combination of such searches excludes a SM-like Higgs boson up to masses of around $m_{H}=600 \mathrm{GeV}$.

Searches targeting specific beyond-SM models are also performed, with particular attention to super-symmetric extensions. A search for a neutral Higgs boson production in association with $b$-quarks has been performed using $4.7 \mathrm{fb}^{-1}$ of data collected in 2011 [15]. The Higgs boson is searched when decaying to a pair of muons or leptons, and events are classified depending if they have or not a $b$-tagged jet. Limits on the production cross section of such a particle are given as model-independent limits on cross section times acceptance as well as in the scalar mass versus $\tan \beta$ plane in the Minimal Super-Symmetric Model (MSSM) framework.

Searches for charged Higgs production [16, 17] are also performed in the $c \bar{s}$ (and charge conjugate) and $\tau^{ \pm} \stackrel{(-)}{v}$ final states, focusing on charge Higgs masses below the top quark mass. Events consistent with $t \bar{t}$ production where one of the top decays to a charged Higgs boson and a $b$-quark are selected; no excess of events is found on top of the expected background and 95\% C.L. limits are set on the cross section times efficiency as well as in the charge particle mass versus $\tan \beta$ plane of the MSSM model.

\section{Conclusions}

Since the discovery of a new boson with a mass around $125 \mathrm{GeV}$ made on July 2012, ATLAS has been actively working on increasing the information available on the new discovered particle. Updated results on the $b \bar{b}$ and $\tau \tau$ decay channels are closed to probe production cross sections comparable to the ones expected from the SM. In addition a vast program of searches for beyond SM signatures of Higgs-like production is being performed, with particular emphasis to neutral and charged Higgs production within the Minimal Super-Symmetric Model, but presenting results in such a way that those limits can be easily interpreted in the framework of other theories. 


\section{References}

[1] ATLAS Collaboration, Observation of a new particle in the search for the Standard Model Higgs boson with the ATLAS detector at the LHC, Phys.Lett. B716 (2012) 1-29 [arXiv:1207.7214].

[2] CMS Collaboration, Observation of a new boson at a mass of $125 \mathrm{GeV}$ with the CMS experiment at the LHC, Phys.Lett. B716 (2012) 30-61 [arXiv:1207.7235].

[3] C. Schmitt (ATLAS Collaboration), Combined Higgs result from ATLAS, Published with this contribution..

[4] LHC Higgs Cross Section Working Group, Handbook of LHC Higgs Cross Sections: 1. Inclusive Observables, CERN-2011-002 [arXiv:1101.0593]

[5] LHC Higgs Cross Section Working Group, Handbook of LHC Higgs Cross Sections: 2. Differential Distributions, [arXiv:1201.3084]

[6] ATLAS Collaboration, The ATLAS experiment at the CERN Large Hadron Collider, JINST 3 S08003 (2008).

[7] ATLAS Collaboration, https://twiki.cern.ch/twiki/bin/view/AtlasPublic/HiggsPublicResults

[8] ATLAS Collaboration, Update of the $H \rightarrow W W^{(\star)} \rightarrow e v \mu v$ analysis with $13.0 \mathrm{fb}^{-1}$ of $\sqrt{s}=8 \mathrm{TeV}$ data collected with the ATLAS detector, ATLAS-CONF-2012-158, [https://cds.cern.ch/record/1493601]

[9] ATLAS Collaboration, Search for the Standard Model Higgs boson in $H \rightarrow \tau^{+} \tau^{-}$decays in proton-proton collisions with the ATLAS detector, ATLAS-CONF-2012-160, [https://cds.cern.ch/record/1493624]

[10] ATLAS Collaboration, Search for the Standard Model Higgs boson produced in association with a vector boson and decaying to bottom quarks with the ATLAS detector, ATLAS-CONF-2012-161, [https://cds.cern.ch/record/1493625]

[11] ATLAS Collaboration, Search for the Standard Model Higgs boson produced in association with top quarks in proton-proton collisions at $\sqrt{s}=7 \mathrm{TeV}$ using the ATLAS detector,

ATLAS-CONF-2012-135, [https://cds.cern.ch/record/1478423]

[12] ATLAS Collaboration, Search for a Standard Model Higgs boson in the $H \rightarrow Z Z \rightarrow \ell \ell v v$ decay channel using $4.7 \mathrm{fb}^{-1}$ of $\sqrt{(s)}=7 \mathrm{TeV}$ data with the ATLAS Detector, ATLAS-CONF-2012-016, [https://cds.cern.ch/record/1429665]

[13] ATLAS Collaboration, Search for a Standard Model Higgs boson in the mass range 200-600 GeV in the $H \rightarrow Z Z \rightarrow \ell \ell q q$ decay channel with the ATLAS Detector, ATLAS-CONF-2012-017, [https://cds.cern.ch/record/1429666]

[14] ATLAS Collaboration, Search for the Higgs boson in the $H \rightarrow W W \rightarrow \ell v j j$ decay channel using 4.7 $\mathrm{fb}^{-} 1$ of pp collisions at $\sqrt{(s)}=7 \mathrm{TeV}$ with the ATLAS detector, ATLAS-CONF-2012-018, [https://cds.cern.ch/record/1429667]

[15] ATLAS Collaboration, Search for neutral MSSM Higgs bosons in $\sqrt{s}=7 \mathrm{TeV}$ pp collisions with the ATLAS detector., ATLAS-CONF-2012-094, [https://cds.cern.ch/record/1460440]

[16] ATLAS Collaboration, Search for charged Higgs bosons decaying via $H \rightarrow \tau v$ in $t \bar{t}$ events using 4.6 $\mathrm{fb}^{-} 1$ of collision data at $\sqrt{(s)}=7 \mathrm{TeV}$ with the ATLAS detector., ATLAS-CONF-2012-011, [https://cds.cern.ch/record/1429659]

[17] ATLAS Collaboration, A search for a light charged Higgs boson decaying to c $\bar{s}$ in pp collisions at $\sqrt{s}=7 \mathrm{TeV}$ with the ATLAS detector, ATLAS-CONF-2011-094, [https://cds.cern.ch/record/1367737] 